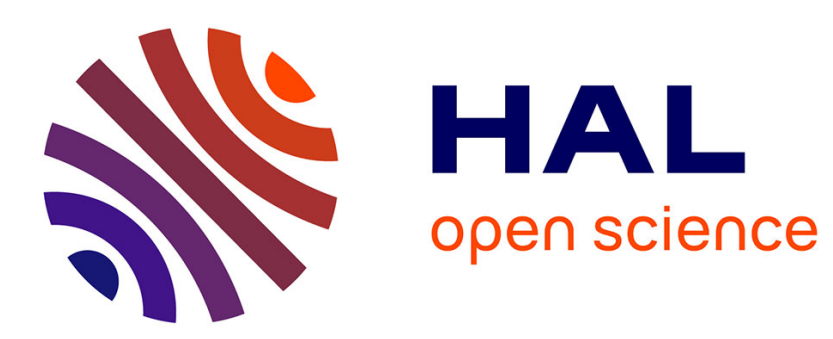

\title{
Computer-aided design and optimization of a redundant robotic system for automated fiber placement process
}

\author{
Divia Shah, Jiuchun Gao, Anatol Pashkevich, Stéphane Caro, Benoit
}

Courtemanche

\section{- To cite this version:}

Divia Shah, Jiuchun Gao, Anatol Pashkevich, Stéphane Caro, Benoit Courtemanche. Computer-aided design and optimization of a redundant robotic system for automated fiber placement process. AIP Conference Proceedings, Jul 2018, Surabaya, Indonesia. 10.1063/1.5046258 . hal-01980737

\section{HAL Id: hal-01980737 https://hal.science/hal-01980737}

Submitted on 14 Jan 2019

HAL is a multi-disciplinary open access archive for the deposit and dissemination of scientific research documents, whether they are published or not. The documents may come from teaching and research institutions in France or abroad, or from public or private research centers.
L'archive ouverte pluridisciplinaire HAL, est destinée au dépôt et à la diffusion de documents scientifiques de niveau recherche, publiés ou non, émanant des établissements d'enseignement et de recherche français ou étrangers, des laboratoires publics ou privés. 


\title{
Computer-Aided Design and Optimization of a Redundant Robotic System for Automated Fiber Placement Process
}

\author{
Divya Shah ${ }^{1,2}$, Jiuchun Gao ${ }^{1,3}$, Anatol Pashkevich ${ }^{1,3}$, Stéphane Caro ${ }^{1,4}$ and Benoît Courtemanche \\ ${ }^{1}$ Laboratoire des Sciences du Numérique de Nantes, 1 Rue de la Noë, Nantes 44300, France. \\ ${ }^{2}$ École Centrale de Nantes, 1 Rue de la Noë, Nantes 44300, France. \\ ${ }^{3}$ Institut Mines-Télécom Atlantique, 4 Rue Alfred-Kastler, Nantes 44307, France. \\ ${ }^{4}$ Centre National de la Recherche Scientifique (CNRS), Nantes, France. \\ ${ }^{5}$ Centre Technique des Industries Mécaniques (CETIM), 74 Route de la Jonelière, Nantes 44000, France. \\ E-mail: divya-haresh.shah@eleves.ec-nantes.fr
}

\begin{abstract}
This paper proposes a comprehensive methodology for the computer-aided design and optimization of a robotic workcell for the automated fiber placement. The robotic cell, comprising of a 6 -axis industrial manipulator and an actuated positioner, is kinematically redundant with respect to the considered task. An efficient optimization technique for managing these kinematic redundancies is proposed. The robot/positioner motion planning and robotic cell layout design in a CAD environment are presented. To confirm validity of the developed methodology, experimental results are presented that deal with automation of thermoplastic fiber placement process.
\end{abstract}

Keywords: Robotic fibre placement; Computer-Aided Design; Optimal motion planning; Collision detection; Robot programming

\section{INTRODUCTION}

Recently, use of fiber reinforced composite materials has drastically increased, mainly in aerospace and automotive industries [1]. Compared to their conventional counterparts, composite materials offer better stiffness-to-weight ratio, strength-to-weight ratio, flexibility of shaping and corrosion resistance [2]. To produce components from composite materials, a new technological process AFP (Automated Fiber Placement) is increasingly implemented. In this process the workpiece molds or liners are mounted on the shaft of a rotating positioner to improve the accessibility of certain locations. An industrial manipulator equipped with a specialized technological tool is used to heat the fiber tows and place them in-situ using a pressure roller [3].

A typical AFP workcell, comprising of a 6-axis manipulator and 1- or 2-axis positioner for rotating the workpiece, has at least 7-8 degrees of freedom (DOF) whereas the fiber placement task requires only 6 DOF. This kinematic redundancy provides the user with some flexibility in programming the robot motions, and at the same time creates a problem of redundancy management in an optimal way in order to maximize the productivity. At present, a general trend in literature for redundancy resolution is based on converting this continuous path problem into a discrete one [4]. Further enhancements were successfully achieved for the applications of laser cutting in [5] following a graph-based search. For fiber placement application, manipulator motion planning was developed in [6] assuming constant tool velocity. An alternative approach was proposed in [7], focusing on tool path smoothing in Cartesian space. In [8], the authors propose a singularity-free optimal design for a 2T2R parallel robot with a 2 -axis positioner, thus eliminating the kinematic redundancy for the AFP process.

Another challenge in implementation of AFP is to design the optimal layout of the robotic system and to automate the robot programming process. Existing works in this area mainly deal with welding applications [9]. For the fiber placement, some results were presented in [10].
However, the complete design and optimization procedure for AFP is very complicated and time-consuming. For this reason, this work concentrates on CAD-based design of workstations for AFP technology taking into account kinematic redundancy of the robotic system.

\section{COMPUTER-AIDED DESIGN OF ROBOTIC CELL}

The developed methodology for the design and optimization a robotic system for AFP is presented in Figure-1. The inputs in this process are the robot, positioner, tool and the workpiece models. The first step is the robotic cell assembly, wherein the layout of workcell is determined, and the manufacturing task is defined considering the desired placement track.

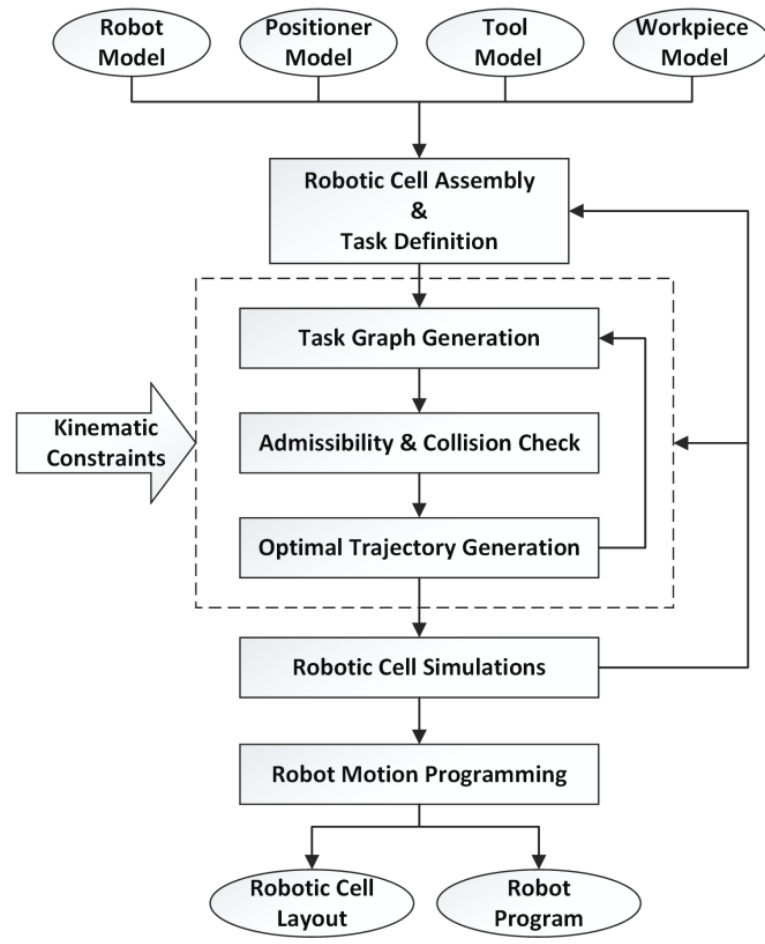

Figure-1. An outline of computer-aided design and optimization methodology for AFP process. 
The next step involves generation of optimal motions for the system taking into consideration the joint limits and kinematic singularities and checking for the admissibility of the relevant locations of the technological tool. At the final steps, the robotic cell simulations and motion programming is performed. If some problems are detected during simulations, the design process can be reconfigured and repeated with necessary modifications in the optimal motion generation or the cell assembly steps. The overall outcomes of the procedure include the cell layout along with robot programs for optimal trajectories, ready to be implemented. Each of these aspects is discussed in detail in the subsequent sections of this paper.

\section{ROBOTIC CELL ASSEMBLY \& TASK DEFINITION}

The computer-aided design of the robotic cell includes selection of the workcell components and their relative positioning. To simplify this process, the robotic CAD system includes a library of commercially available robots and auxiliary devices. The positioning of the components is adjusted in a manner that allows the robot to reach the entire surface of the workpiece. In addition, certain CAD systems also include specialized tools allowing the user to create a 3D model of the workpiece and generate the fiber placement tracks. This workpiece model is also an input for robotic cell design process.

\section{D Modeling of Robotic Cell}

The robotic system considered in this work consists of 6-axis serial anthropomorphic robot, specialized placement tool, 1-axis rotating positioner and a 1 -axis linear track. Thus the system has $8 \mathrm{DOF}$, with a degree of redundancy equal to 2. Depending upon the geometry of the workpiece in consideration, the robot base location on the linear track can be fixed or changed online; thus providing the additional flexibility.

Using the standard step/iges files available from the manufacturer's catalogs, each of the components was designed in the CATIA V5 software from Dassault Systems as shown in Figure-2. All the active joints were defined so as to be able to simulate the system using joint coordinates.

\section{Kinematic Modeling of Robot \& Positioner}

For developing the kinematic model of the system, a frame is attached to each component of the robotic cell as follows:

- $\boldsymbol{F}_{\mathbf{0}}$ : Global frame with Z-axis in the vertical direction.

- $\boldsymbol{F}_{\boldsymbol{R} \boldsymbol{B}}$ : Robot base frame with origin at the base of the robot and orientation same as the global frame.

- $\boldsymbol{F}_{\boldsymbol{P}}$ : Positioner frame with origin at the center of rotating flange and $\mathrm{Z}$-axis as the rotating axis.

- $\boldsymbol{F}_{\boldsymbol{W}}$ : Workpiece frame with Z-axis along the direction of the workpiece liner.

- $\quad \boldsymbol{F}_{T}$ : Tool frame located at Tool Center Point (TCP).

- $\boldsymbol{F}_{\boldsymbol{L}}$ : Linear track frame with origin at the centre of the moving rail and orientation same as global frame.

The geometric model for the robot $g_{r}$ is obtained using the standard Denavit-Hartenberg parameters and presented in the following way:

$g_{r}\left(\mathbf{q}_{r}\right)=\prod_{j=1}^{6}{ }^{j-1} \mathbf{T}_{j}\left(q_{j}\right)$,

where, $\mathbf{T}$ is the $4 \times 4$ homogenous transformation matrix, $\mathbf{q}_{r}$ are the joint coordinates and $j$ is the joint index. Similarly, the geometric model for the positioner $g_{p}$ is presented as a $4 \times 4$ homogenous rotation matrix $\operatorname{Rot}_{Z}$ around the Z-axis;

$g_{p}\left(q_{p}\right)=\operatorname{Rot}_{Z}\left(q_{p}\right) ;$

and for the linear track $g_{l}$ as a $4 \times 4$ homogenous translational matrix Trans $_{X}$ along the $\mathrm{X}$-axis;

$g_{l}\left(q_{l}\right)=\operatorname{Trans}_{X}\left(q_{l}\right)$.

\section{Manufacturing Task Definition}

In the developed system, there are some specialized tools to define fiber placement tracks for the desired workpiece. These tracks are then discretized into a set of points, called task locations, expressed in the workpiece frame. A Cartesian coordinate frame $\boldsymbol{F}_{\boldsymbol{T} \boldsymbol{L}}$ is attached to each of the task location such that the $\mathrm{Z}$-axis is always normal to the workpiece surface and the $\mathrm{X}$-axis follows the travelling direction of the track. The following equation represents the $n$ task locations, indexed as $i$;

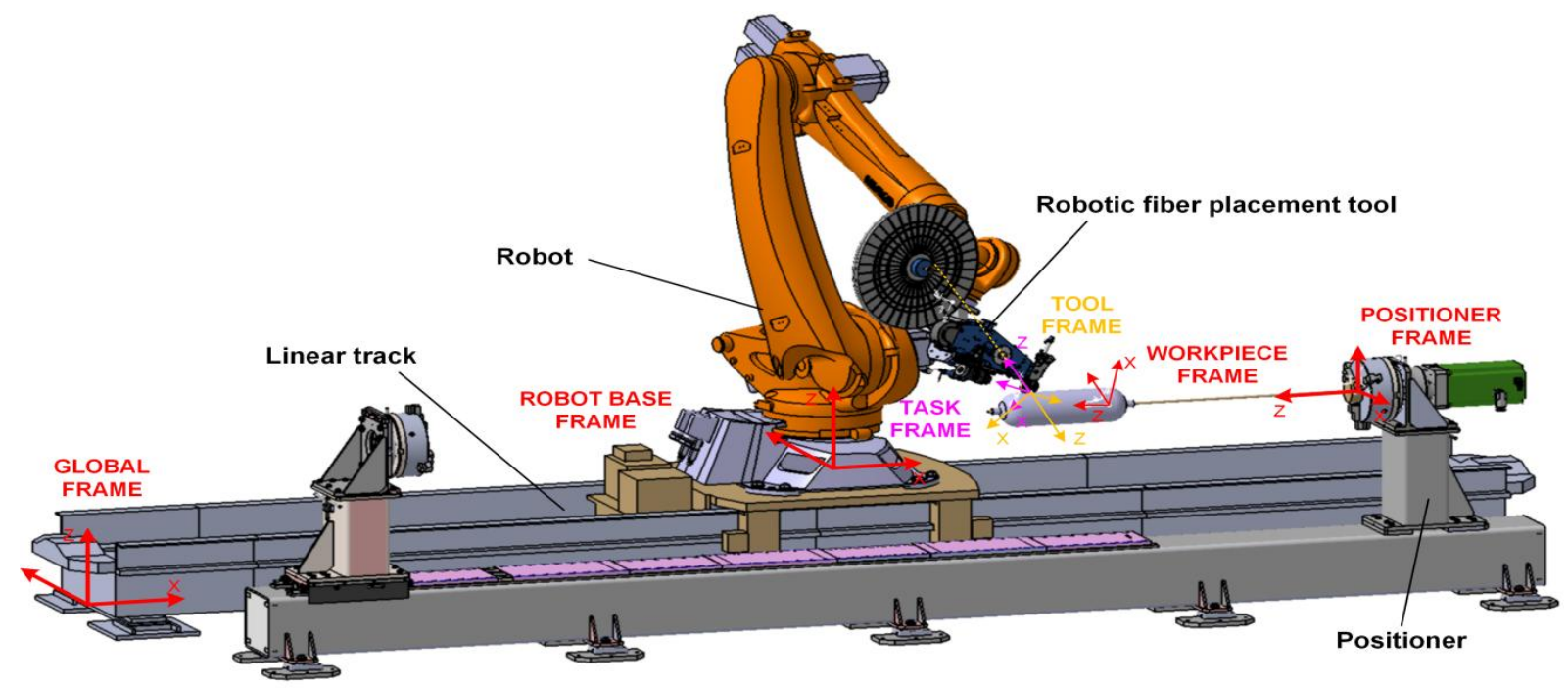

Figure-2. CAD model of the AFP robotic workcell 
as $4 \times 4$ homogenous transformation matrices $\mathbf{T}$ expressed in the workpiece frame;

$$
{ }^{W} \mathbf{T}_{T L_{i}}=\left[\begin{array}{cccc}
\mathbf{n}_{\mathbf{i}} & \mathbf{s}_{\mathbf{i}} & \mathbf{a}_{\mathbf{i}} & \mathbf{p}_{\mathbf{i}} \\
0 & 0 & 0 & 1
\end{array}\right] ; \forall i=1,2, \ldots, n,
$$

where $\mathbf{n}_{\mathbf{i}}, \mathbf{s}_{\mathbf{i}}$ and $\mathbf{a}_{\mathbf{i}}$ are the unit vectors along the respective Cartesian axes $\mathrm{X}, \mathrm{Y}$ and $\mathrm{Z}$ forming the orientation matrix. The vector $\mathbf{p}_{\mathbf{i}}$ represents the position vector of the individual task location in the same Cartesian frame. These set of points and the related frames constitute the 'task model', represented in the Cartesian space.

\section{OPTIMAL TRAJECTORY GENERATION}

For the considered AFP process, where the tool speed variations are allowed, a discrete optimization based methodology was proposed in [11] to generate timeoptimal trajectories considering one degree of redundancy, where the robot base location on the linear track is fixed. They are summarized below.

\section{Task Graph Generation}

Consider the time interval $t \in[0, T]$ and the sequence of time instants $\left\{t_{1}, t_{2}, \ldots, t_{n}\right\}$ corresponding to each of the task locations defined in eqn. (4). Let us also, define the functions $\mathbf{q}_{\mathbf{r}}(t)$ and $q_{p}(t)$, describing the robot and the positioner motions respectively. The optimization problem can thus be formulated as minimizing the total travelling time $T$. This problem is subject to the following equality constraint representing the closed loop form of the system:

$$
{ }^{0} \mathbf{T}_{R B} \cdot g_{r}\left(\mathbf{q}_{r}\right) \cdot{ }^{R F} \mathbf{T}_{T} \cdot{ }^{T} \mathbf{T}_{T L_{i}}={ }^{0} \mathbf{T}_{P} \cdot g_{P}\left(q_{p}\right) \cdot{ }^{P} \mathbf{T}_{W} \cdot{ }^{W} \mathbf{T}_{T L_{i}},
$$

where all notations are defined in previous section.

It is clear that this system is nonlinear and redundant. Here, it is reasonable to treat the positioner coordinate as the redundant variable, which allows using robot and positioner models independently. This redundant variable is sampled with a step of $\Delta q_{p}$ as follows:

$q_{p}^{(k)} \in\left[q_{p}^{\min }, q_{p}^{\max }\right]$

where $k=0,1, \ldots, m$ and $m=\left(q_{p}^{\max }-q_{p}^{\min }\right) / \Delta q_{p}$. Then, by computing the positioner direct kinematics and robot inverse kinematics as given by eqn. (7); $\boldsymbol{\mu}$ being the robot configuration index vector:

$$
\mathbf{q}_{r}^{(k)}\left(t_{i}\right)=g_{r}^{-1}\left(g_{p}\left(q_{p}^{(k)}\left(t_{i}\right)\right), \boldsymbol{\mu}\right) ;
$$

a set of all possible robot and positioner configurations for every sampling step of the positioner coordinate and for every task location of the given placement track, can be obtained:

$$
\mathbf{L}_{T L}^{(k, i)}=\left(\mathbf{q}_{r}^{(k)}\left(t_{i}\right), q_{p}^{(k)}\left(t_{i}\right)\right) .
$$

These 'location cells', expressed in the joint space, form the 'task graph' as shown in Figure-3.

In case of two degrees of redundancy, similar sets of location cells can be computed for every sampling step of the linear axis coordinate $q_{l}$, thus generating a twodimensional task graph.

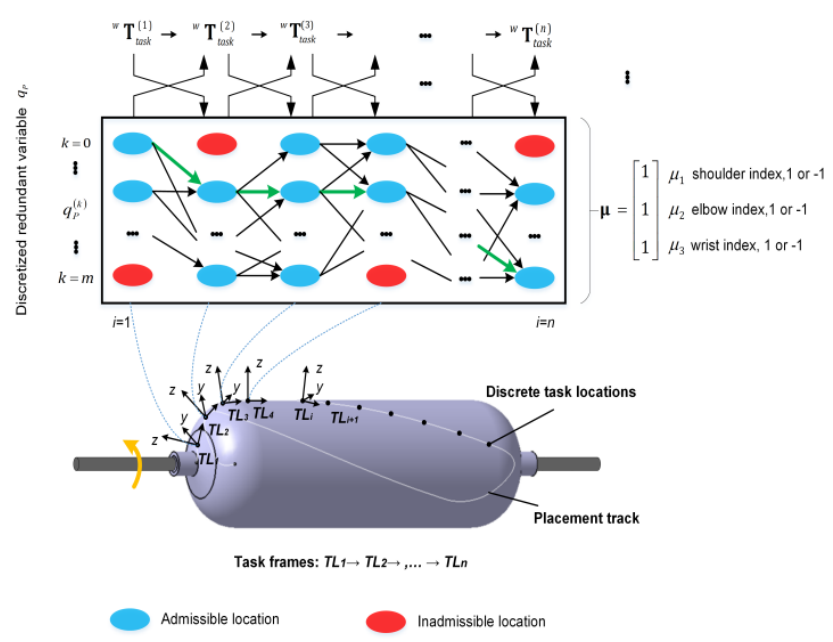

Figure-3. Task-graph representation.

\section{Admissibility \& Collision Check}

The next step is to verify the admissibility of these location cells on the task graph, i.e., to check for the practical feasibility of the configuration represented by the cell against the constraints. The cells violating any of these constraints are flagged "inadmissible".

Firstly, the task graph is checked for the actuator position, velocity and acceleration limits and the kinematic singularities of the robot, as given by the following equation:

$q_{j}^{\min } \leq q_{j}\left(t_{i}\right) \leq q_{j}^{\max }$

$\dot{q}_{j}^{\min } \leq \dot{q}_{j}\left(t_{i}\right) \leq \dot{q}_{j}^{\max }$,

$\ddot{q}_{j}^{\min } \leq \ddot{q}_{j}\left(t_{i}\right) \leq \ddot{q}_{j}^{\max }$

where $j$ represents all actuator joints (robot and positioner).

The task graph, thus reduced, is then simulated on the CAD model to detect for collisions within the workcell. Various interferences among the workcell components are defined in the model and when the collisions occur, the colliding components are highlighted, as shown in Figure-4. A set of numerical values are obtained from the CAD model for each location cell, where zero represents all the collision-free candidates, thus obliging the following collision constraint:

$$
\operatorname{cols}\left(\mathbf{q}_{\mathbf{r}}(t), q_{p}(t)\right)=0 ; \forall t \in[0, T] \text {. }
$$

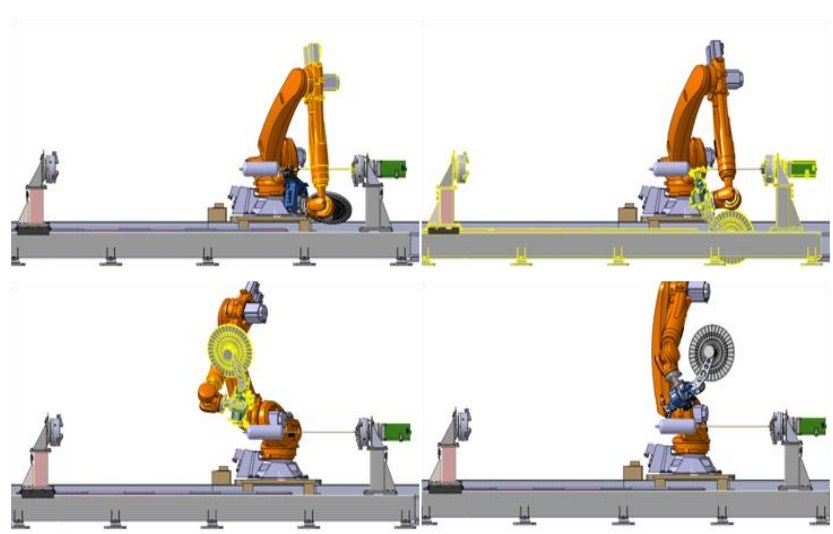

Figure-4. Collision detection in CAD model. 


\section{Optimal Trajectory Planning}

To find the optimal trajectory, the edges on the task graph are weighted according to the minimum travelling time required between consequent locations, restricted by the maximum actuator velocities and accelerations. The objective function (robot processing time) can now be presented as a summation of these edge weights, expressed as dist :

$T=\sum_{i=1}^{n-1} \operatorname{dist}\left(\mathbf{L}_{T L}^{\left(k_{i}, i\right)}, \mathbf{L}_{T L}^{\left(k_{i+1}, i+1\right)}\right)$,

The optimization algorithm is based on the dynamic programming principle to compute the "shortest" path, with its length denoted as $d_{k, i}$ :

$$
d_{k, i+1}=\min _{k^{\prime}}\left\{d_{k^{\prime}, i}+\operatorname{dist}\left(\mathbf{L}_{T L}^{k, i+1}, \mathbf{L}_{T L}^{k^{\prime}, i}\right)\right\},
$$

These distances are computed sequentially from the second location until the last. The indices $k$ record the optimal solution, expressed in the joint space.

\section{ROBOT CELL SIMULATIONS \& PROGRAMMING}

Debugging and analysis of the optimal robot motions is done by simulating them in the CAD environment. If the results are not acceptable, the procedure will be easily reiterated using task graphs with finer sampling step or even adjustments in the layout of the cell. The robot program for implementation is then generated.

Table-1. Comparison of robot motion commands

\begin{tabular}{|c|c|c|c|}
\hline & PTP & LIN/CIR & SPL \\
\hline Interpolation & Joint-space & Cartesian-space & Both \\
\hline Movement & Start-Stop & Start-Stop & Continuous \\
\hline Time Setting & No & No & Yes \\
\hline
\end{tabular}

A brief comparison among the standard robot motion commands is shown in Table-1, the choice of which is critical to the performance of the system. This is because the travelling time is recomputed by the robot controller based on the motion commands. Hence, with execution of a set of polynomials and the possibility of adjusting the travelling time between nodes, Spline (SPL) commands are favorable.

\section{EXPERIMENTAL RESULTS}

To demonstrate the efficiency of the methods proposed above, the industrial case of fabricating a cylindrical pressure vessel with spherical domes (as shown in Figure-3) using thermoplastic composites was studied in collaboration with Centre Technique des Industries Mécaniques (CETIM). The laser assisted robotic fiber placement platform comprises of the following:

- 6-axis serial manipulator KUKA KR210 R3100 ultra.

- 1-axis linear track KUKA KL 2000.

- 1-axis rotating positioner AFPT 550 series.

- Specialized placement tool from AFPT.
Starting from the individual models, the cell components were assembled together as shown in the Figure-2, such that the rotating axis falls within maximum area of robot workspace. Here, the length of the workpiece in consideration is much shorter than the horizontal reach of the robot. Thus, the robot base location on the linear axis was fixed. The best position was estimated by computing the solutions for different coordinates on the linear axis and comparing the total travelling times of the process, as shown in Figure-5. This provides the cell layout for the considered case.

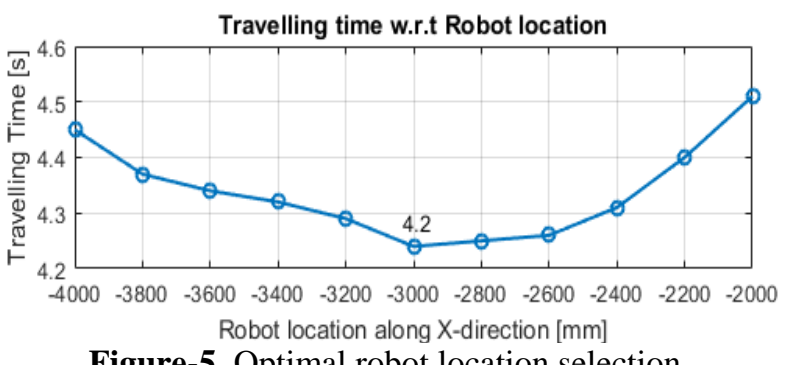

Figure-5. Optimal robot location selection.

The desired placement track is represented by the white curve on the workpiece liner in Figure-3. To fabricate the complete pressure vessel, similar placement tracks are repeated with offsets on the rotating axis, so as to cover the entire surface area. This track was discretized into 200 task locations represented in the Cartesian space referenced to the workpiece frame. The positioner coordinate was sampled for every $1^{\circ}$ and the solutions were generated for each to form the task graph. Following the admissibility check and trajectory planning, an optimal trajectory was generated for the process with the total travelling time $T \cong 3.1 \mathrm{sec}$

The following Figures- 6 and -7 represent the positioner and robot joint coordinate and velocity curves respectively, plotted against the travelling time. The trajectory was simulated in the CAD model and the corresponding robot program, ready to be implemented on the industrial platform, was generated using the SPL commands. Compared to the initial value of $T \cong 14 \mathrm{sec}$, this method reduces the total travelling time of the process by $75 \%$.

This method can be adopted in fabrication of components with various shapes and sizes and complex placement track profiles. Smooth trajectories can be obtained by finer discretization of the placement track and finer sampling of the external axes. The use of the linear axis online allows for fabrication of long workpieces.

\section{CONCLUSIONS}

This paper contributes towards the complete methodology for computer-aided design and optimization of a redundant robotic system for the automated fiber placement problem. It integrates the computational tools for optimal motion planning with the CAD tools for design, simulation and verification. The theoretical results obtained in this work promise significant reduction in the processing time, thus improving the overall efficiency. Future work will involve the implementation of these results on the industrial platform at CETIM. 

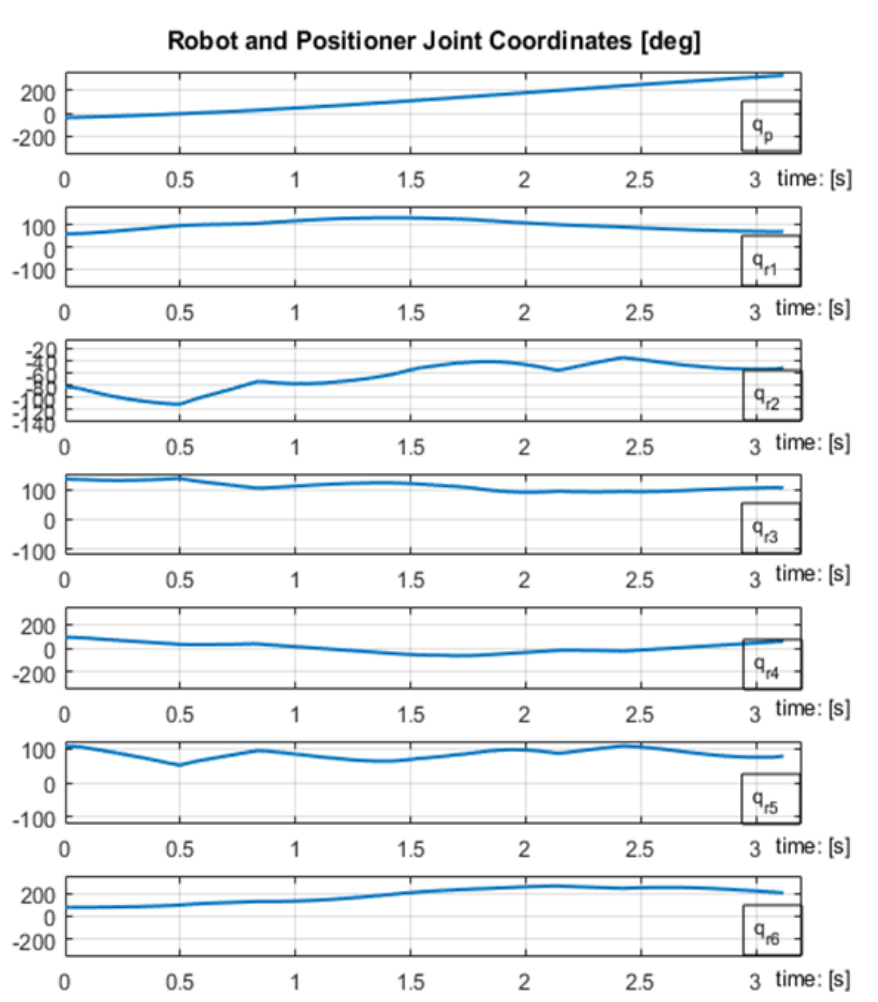

Figure-6. Robot and Positioner Joint Coordinates.

\section{REFERENCES}

[1] G. Marsh, "Automating aerospace composites production with fibre placement," Reinf. Plast., vol. 55, no. 3, pp. 32-37, 2011.

[2] D. Gay, Composite materials: design and applications. CRC press, 2014.

[3] S. T. Peters, Handbook of composites. Springer Science \& Business Media, 2013.

[4] L. Li, D. Xu, X. Wang, and M. Tan, “A survey on path planning algorithms in robotic fibre placement," in Control and Decision Conference (CCDC), 2015 27th Chinese, 2015, pp. 4704-4709.

[5] A. Dolgui and A. Pashkevich, "Manipulator motion planning for high-speed robotic laser cutting," Int. J. Prod. Res., vol. 47, no. 20, pp. 5691-5715, 2009.

[6] T. Martinec, J. Mlýnek, and M. Petrů, "Calculation of the robot trajectory for the optimum directional orientation of fibre placement in the manufacture of composite profile frames," Robot. Comput. Integr. Manuf., vol. 35, pp. 42-54,
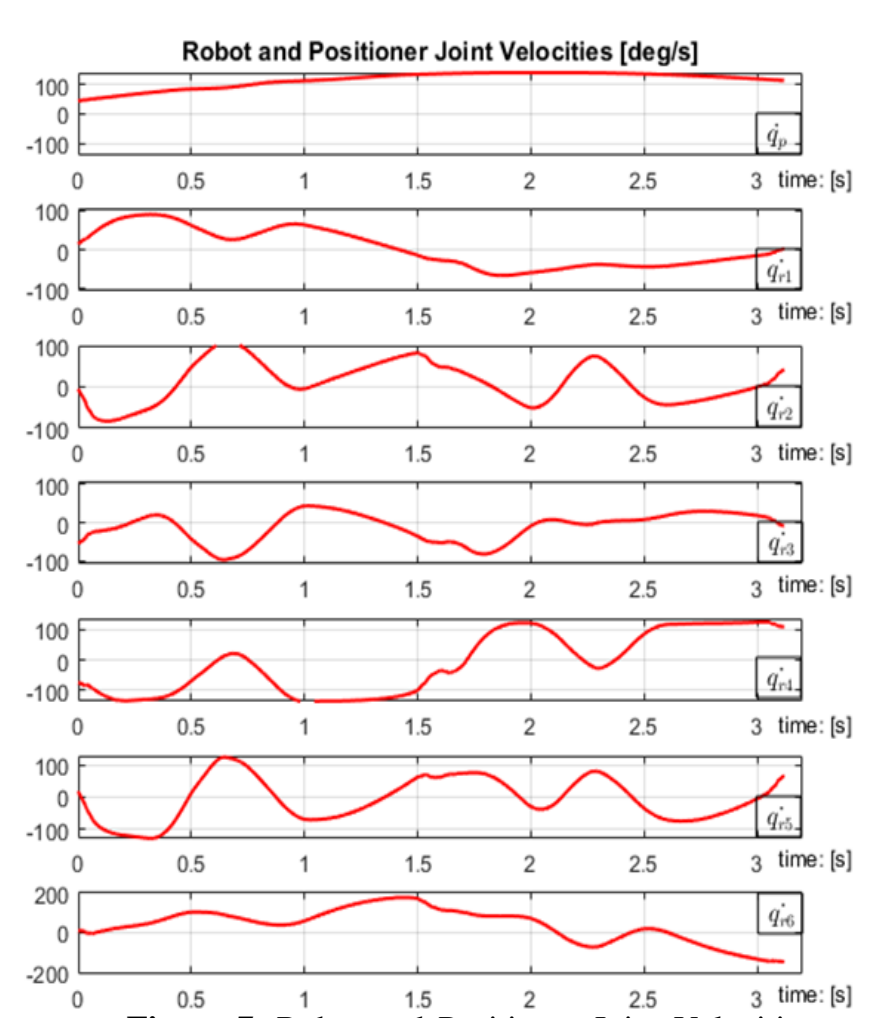

Figure-7. Robot and Positioner Joint Velocities.

2015.

[7] P. Debout, H. Chanal, and E. Duc, "Tool path smoothing of a redundant machine: Application to Automated Fiber Placement," CAD Comput. Aided Des., 2011.

[8] D. Gan, J. S. Dai, J. Dias, R. Umer, and L. Seneviratne, "Singularity-Free Workspace Aimed Optimal Design of a 2T2R Parallel Mechanism for Automated Fiber Placement," J. Mech. Robot., vol. 7, no. 4, p. 41022, 2015.

[9] X. Li, B. Cheng, C. Li, H. Zhang, and Y. Li, "Seeder Rack Welding Robot Workstation Design Based on DELMIA," in Industrial Engineering and Engineering Management 2015: Core Theory and Applications of Industrial Engineering (Vol. 1), 22nd International Conference on, 2016, pp. 643-654.

[10] C. Krombholz and M. Perner, "ADVANCED AUTOMATED FIBRE PLACEMENT,” 2013.

[11] J. Gao, A. Pashkevich, and S. Caro, "Optimization of the robot and positioner motion in a redundant fiber placement workcell," Mech. Mach. Theory, vol. 114, pp. 170-189, 2017. 